\title{
Unilateral Sensorimotor Cortex Lesions in Adult Rats Facilitate Motor Skill Learning with the "Unaffected" Forelimb and Training- Induced Dendritic Structural Plasticity in the Motor Cortex
}

\author{
Scott D. Bury ${ }^{1}$ and Theresa A. Jones ${ }^{2}$ \\ ${ }^{1}$ Department of Psychology, University of Washington, Seattle, Washington 98195, and ${ }^{2}$ Department of Psychology and \\ Institute for Neuroscience Research, University of Texas, Austin, Texas 78712
}

In humans and other animals, sufficient unilateral damage to the sensorimotor cortex can cause impairments in the opposite forelimb and the development of a hyper-reliance on the nonimpaired limb. This hyper-reliance is adaptive to the extent that it contributes to functional compensation for lesion-induced impairments. We have found that unilateral lesions of the forelimb region of the sensorimotor cortex (FLsmc) in rats, or callosal transections, cause neurons of the opposite motor cortex to become exceptionally responsive to changes in forelimb behavior. This enhanced responsiveness might facilitate learning of compensatory strategies with the nonimpaired forelimb after unilateral FLsmc lesions. The possibility that these lesions facilitate learning with the nonimpaired forelimb was addressed in this study. Rats were required to learn a skilled forelimb reaching task after either unilateral FLsmc lesions or sham operations. The trained limb in animals with lesions was the nonimpaired limb. Compared with shams, rats with unilateral lesions had a greater rate of acquisition and asymptotic performance level on the task, which was especially evident on more difficult trials. Quantitative measures of microtubule associated protein-2 (MAP2) immunostained dendrites indicated an enhancement of training-induced dendritic cytoskeletal changes in the motor cortex opposite lesions. Thus, unilateral FLsmc lesions facilitate learning of at least some types of motor skills using the nonimpaired forelimb as well as some of the neuronal changes associated with this learning. This facilitation could be a substrate underlying behavioral compensation for unilateral FLsmc damage and may contribute to the phenomenon of learned nonuse of the impaired limb.

Key words: learned nonuse; behavioral compensation; reach training; denervation; dendritic growth; rehabilitative training; microtubule associated protein-2
Research on the neural events after cortical damage in adult animals has demonstrated that neural growth and restructuring is influenced by postinjury behavioral changes (Jones et al., 1998). Unilateral damage to the forelimb representation area of the sensorimotor cortex (FLsmc) in adult rats produces pronounced sensorimotor deficits in the contralateral forelimb and a compensatory hyper-reliance on the ipsilateral, or nonimpaired, forelimb (Jones and Schallert, 1992). In the motor cortex opposite the lesions, there are time-dependent cellular and structural changes, including increases in astrocytic neurotrophic factor expression, dendritic arborizations, and synapse number per neuron (Jones and Schallert, 1992; Jones et al., 1996; Dahms et al., 1999; Jones, 1999). The neuronal changes were found to be dependent on forelimb behavioral changes (Jones and Schallert, 1994) and were enhanced by training on a complex motor skills task (Jones et al., 1999). However, manipulations in intact rats that peripherally mimicked the behavioral effects of the lesion were not sufficient to reproduce in magnitude and time course the neuronal changes

Received April 26, 2002; revised July 11, 2002; accepted July 16, 2002.

This study was supported by National Institutes of Health Grants MH56361 and MH64586. We thank Dr. Jeffery Kleim for his advice and guidance concerning reach training, Dr. Timothy Schallert for his comments concerning the behavioral analyses, Dr. Ilene Bernstein for the use of her microscopes, DeAnna Adkins for help with the MAP2 procedures, and Kevin McCullough and Jurate Lasiene for their help with training and collection of data.

Correspondence should be addressed to Dr. Scott D. Bury, Reeve-Irvine Research Center, University of California-Irvine, 2216 Gillespie Neuroscience Research Facility, Irvine, CA 92697-4292. E-mail: sbury@uci.edu.

Copyright (C) 2002 Society for Neuroscience $0270-6474 / 02 / 228597-10 \$ 15.00 / 0$ found after cortical damage (Jones and Schallert, 1994; Adkins et al., 2002)

These findings led to the hypothesis that lesion-induced degenerative events can facilitate behaviorally induced neuronal change. Research using independent manipulations of motor cortical denervation (callosal transections) and forelimb behavioral change (forced forelimb use) supports this hypothesis. Animals with transections of the corpus callosum in combination with forced forelimb use had extensive increases in the dendritic arborization (Bury et al., 2000a) and spine number (Adkins et al., 2002) of layer $\mathrm{V}$ pyramidal neurons in the motor cortex opposite the forced-use limb. In contrast, animals that received forced-use or denervation alone showed no dendritic growth and only subtle spine changes in comparison with controls.

Thus, it appears that motor cortical neurons that have been partially denervated of transcallosal projections have an enhanced propensity to grow dendrites and spines in response to forelimb behavioral change. However, it had not yet been determined whether this enhanced neuronal response corresponds to an enhanced capacity to learn new forelimb motor skills. The purpose of these experiments was to determine whether unilateral FLsmc lesions could facilitate learning of a motor skills task with the nonimpaired forelimb and whether this is reflected in greater dendritic changes in the motor cortex. Adult rats were given either unilateral electrolytic FLsmc lesions or sham operation procedures. After surgeries, rats were then trained on a skilled unilateral forelimb reaching task. The hypothesis was that animals with unilateral FLsmc lesions would demonstrate a greater acquisition rate and asymptotic performance level using 
their nonimpaired forelimb in comparison with shams. Additional manipulations were performed to determine the dependency of these effects on preoperative task experience and endogenous limb preferences. Quantitative measures of dendritic processes immunoreactive for microtubule associated protein-2 (MAP2), a structural MAP localized to neuronal dendrites and somata (Kobayashi and Mundel, 1998), were used to detect dendritic alterations in the motor cortex resulting from training of the opposite forelimb, from lesions of the opposite cortex, and from the combination of the two.

\section{MATERIALS AND METHODS}

\section{Subjects and overview of experimental designs}

Fifty-two male Long-Evans hooded rats between the ages of 3 and 4 months of age were used. Rats were made tame by frequent handling beginning at $\sim 2$ months of age and were housed in pairs in transparent tub cages, receiving food and water ad libitum. All animal use was in accordance with a protocol approved by the Animal Care and Use Committee of the University of Washington.

Twelve rats ( $n=6$ unilateral FLsmc lesions; $n=6$ sham operates) were used in experiment 1 . In this experiment rats received extensive $(20$ d) preoperative training on a unilateral skilled reaching task with the limb they preferred to use for the task. Postoperative training was with the nonpreferred forelimb (which was the nonimpaired limb in FLsmc lesion rats). Thus, in experiment 1 , rats had learned all aspects of the task before surgery except for the performance of the task with the nonpreferred limb.

Forty ( $n=20$ lesions; $n=20$ shams) animals were used in experiment 2, which was similar in behavioral design to the first experiment except that no extensive preoperative training was used. Animals were assigned to one of the following groups: (1) unilateral FLsmc lesion and reach training (Lesion + Training), (2) unilateral FLsmc lesion with no training (Lesion + No-Training), (3) sham operations and reach training (Sham + Training), and (4) sham operations with no reach training (Sham + No-Training). In this experiment, most reach-trained animals ( $n=8$ shams; $n=8$ lesions) received postoperative training using the nonpreferred limb. However, to assess the generalizability of the experimental findings to the preferred limb, a subset of animals $(n=4$ sham; $n=4$ lesion) received postoperative preferred limb training. As in experiment 1 , the trained limb in all animals with unilateral FLsmc lesions in experiment 2 was the forelimb ipsilateral to the lesion, i.e., the nonimpaired forelimb. Another subset of rats ( $n=8 \mathrm{sham} ; n=8$ lesions) received no postsurgical training and served as control groups for the effects of the training and lesions on MAP2 immunoreactivity in the motor cortex and on behavioral outcome measures. Rats were randomly assigned to groups with the exception that animals undergoing postoperative reach training were matched for strength and side of limb preferences and for preoperative task performance.

\section{Surgical methods}

Rats were anesthetized with Equithesin $(150 \mathrm{mg} / \mathrm{kg}$ chloral hydrate and $34 \mathrm{mg} / \mathrm{kg}$ pentobarbitol). Atropine sulfate $(0.1 \mathrm{mg} / \mathrm{kg})$ was used before and after the operation to counteract the respiratory depressive effects of Equithesin. The skull and dura were removed unilaterally between 0.5 $\mathrm{mm}$ posterior and $1.5 \mathrm{~mm}$ anterior to bregma and between 3.0 and 4.5 $\mathrm{mm}$ lateral to midline. A noninsulated platinum electrode was lowered to a depth of $1.7 \mathrm{~mm}$ below dura, and $1 \mathrm{~mA}$ anodal constant current was delivered for $120 \mathrm{sec}$. As the current was being applied, the electrode was moved in eight equally spaced horizontal traverses ( $15 \mathrm{sec}$ each) through the exposed cortex. Sham-operated rats received all procedures up to, but not including, skull removal. Previous research has demonstrated that skull removal results in behavioral and neurochemical asymmetries (Adams et al., 1994).

\section{Behavioral methods}

Reach-training apparatus. Animals were trained in a clear Plexiglas box $(25 \mathrm{~cm}$ long $\times 25 \mathrm{~cm}$ high $\times 15 \mathrm{~cm}$ wide) (Fig. $1 A)$ adapted from apparatuses used by Peterson and Devine (1963), Withers and Greenough (1989), Miklyaeva and Whishaw (1996), and McKenna and Whishaw (1999). In the center of the front wall was a 1-cm-wide window that extended from the floor of the apparatus to a height of $20 \mathrm{~cm}$. A $15-\mathrm{cm}$-long by 5 -cm-wide Plexiglas shelf was attached to the outer front
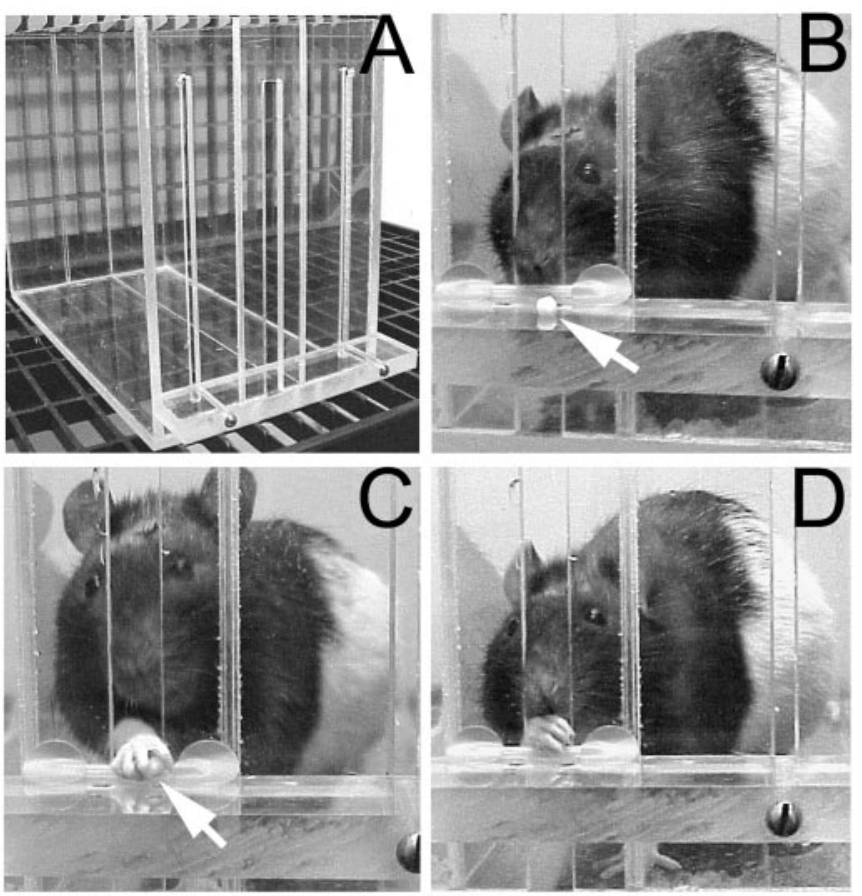

Figure 1. Reach training apparatus $(A)$, and sequential photographs of a rat approaching $(B)$, grasping $(C)$, and retrieving and beginning to eat (D) a food pellet (arrows). An inner chamber wall is to the animal's right.

wall at a height of $3 \mathrm{~cm}$ above the floor of the box. Pellets (45 or $90 \mathrm{mg}$ banana-flavored food pellets; Bioserve Inc., Frenchtown, NJ) were placed in shallow wells in the shelf that were aligned with each vertical edge of the window at a distance of either $1 \mathrm{~cm}$ (close) or $2 \mathrm{~cm}$ (remote) from the outside of the front wall (four wells total). A small piece of plastic tubing $(\sim 2 \mathrm{~mm}$ in diameter) was adhered to the shelf in front of the reaching window. This tubing prevented rats from simply scraping the pellet into their mouth and forced them to grasp the pellet in their paw and lift it over the tubing. To guide reaching with a specific forelimb, pellets were placed in the wells opposite this limb and a removable Plexiglas wall was placed $1.5 \mathrm{~cm}$ from the outer edge of the reaching slot ipsilateral to the trained limb.

Reach-training procedures. Animals were placed on a restricted diet ( $\sim 15 \mathrm{gm} / \mathrm{d}) 1 \mathrm{~d}$ before shaping. Animals were reduced to $90-95 \%$ of their starting weight and maintained at this level throughout the experiment. The rats were initially given a brief shaping period (typically 2-5 d) during which they were placed in the box for $20 \mathrm{~min}$ without the interior wall and with food pellets placed on both sides of the reaching slot at a distance of $1 \mathrm{~cm}$ from the window. In unilateral reaching tasks, nearly all rats develop a preference for using one forelimb for the task (Whishaw, 1992). After rats had clearly demonstrated a preferred limb (defined as the limb used for $>70 \%$ of initial reaches) and were consistently reaching $(>15$ reaches in $20 \mathrm{~min})$ for the pellets, rats began training (experiment 1) or were given surgical procedures (experiment 2 ). Rats that did not meet the $70 \%$ criterion were not used in these studies $(n=5)$. Training periods consisted of $20 \mathrm{~min}$ or 60 single-pellet trials, whichever came first. For each reaching trial, a single pellet was placed in a well, and rats were permitted up to five reach attempts until the pellet was either retrieved or knocked from its well. After each trial, a single food pellet was dropped at the back of the cage to "reset" the rat, to prevent frantic, undirected reaching, and to permit the experimenter to place a new pellet in the well. Performance was measured as the percentage of successful reaches of the total number of reaches attempted (successful reaches + missed reaches + dropped pellets). A successful reach was one in which the rat grasped the pellet from the well and brought it to its mouth and ate it (Fig. $1 B-D)$. Misses include reaches that did not contact the pellet or knocked the pellet from the well. Drops were considered to be reaches in which the pellet was grasped and removed from the well, but dropped before the rat could place it in its mouth. To train a specific limb, the interior wall and pellet placement were manipulated, preventing successful reaches with the nonspecified 
forelimb. Task difficulty was increased by moving the pellet from the $1 \mathrm{~cm}$ distance to $2 \mathrm{~cm}$ from the reaching window. Pellet size was also increased from 45 to $90 \mathrm{mg}$. (Larger pellets were suspected to be more difficult to grasp.)

In experiment 1 , animals were trained for $20 \mathrm{~d}$ presurgically on their preferred forelimb with $45 \mathrm{mg}$ pellets in the close $(1 \mathrm{~cm})$ position. The internal wall and pellet placement were arranged to facilitate reaching with the preferred forelimb. FLsmc lesions were made opposite the preferred limb. After surgery, animals that had lesions or sham operations were then trained with the nonpreferred limb for $18 \mathrm{~d}$. The animals were trained on close pellet trials for $5 \mathrm{~d}$, and then pellets were placed in the remote $(2 \mathrm{~cm})$ position for $8 \mathrm{~d}$. Finally, pellet size was increased from 45 to $90 \mathrm{mg}$, and the pellet was placed in the remote position for the remainder of training $(5 \mathrm{~d})$. In experiment 2 , animals received preoperative shaping and pretraining procedures (to determine endogenous limb preferences) but were not given further training with their preferred forelimb. Rats were allowed to recover for $2 \mathrm{~d}$ after surgery and then trained on close, $45 \mathrm{mg}$ pellet trials for $6 \mathrm{~d}$. Beginning on day 7 and for the remainder of the training, close and remote pellet trials were administered in blocks of 30 trials each (1 block of each per day). The order of close and remote pellet trial blocks was alternated daily. Because it did not result in a major change in performance in experiment 1 , the larger-sized pellet was not used in experiment 2. In all experiments, the postoperatively trained limb in rats with FLsmc lesions was the nonimpaired limb.

Assessments of sensorimotor function. Rats from experiment 2 were used to assess whether training of the nonimpaired limb after FLsmc lesions affects functional outcome in comparison with animals that received no postlesion training. Sensorimotor tests were administered before surgery ("day 0") and on several postoperative days. The footfault test was used as a measure of coordinated forelimb use. This test requires rats to traverse an elevated grid of bars for $2 \mathrm{~min}$ while forelimb misplacements, revealed as slips through the grid openings, are recorded. This test has been reported to be sensitive to lesion-induced impairments in coordinated limb placement during locomotion (Barth et al., 1990). It is not intended to be a sensitive measure of motor learning. The test was administered on day 0 and on postoperative days 2,8 , and 18 . On day 19 , a variation of the footfault test, the lidocaine challenge, was administered. This tests the compensatory reliance on the nonimpaired forelimb by peripherally anesthetizing it while rats are traversing the grid (Schallert et al., 1997). In animals with unilateral FLsmc lesions, this test has been shown to reinstate severe footfaults in the impaired forelimb of animals that appeared completely recovered on the task when permitted to use both forelimbs (for review, see Schallert et al., 1997). Axillary injections of $0.1 \mathrm{cc}$ of $1 \%$ lidocaine were made into the radial nerve region of the nonimpaired, which was also the reach-trained forelimb of the rats immediately before their placement on the footfault test apparatus.

In addition to the footfault test, rats were administered tests of forelimb asymmetries in postural support behavior [the cylinder test (Schallert et al., 1983)] as well measures of responsiveness to somatic-sensory stimulation [the bilateral tactile stimulation test (Schallert and Whishaw, 1984)]. These data are not shown because they revealed lesion-induced impairments in the forelimb that were similar to previously reported findings of the effects of unilateral FLsmc lesions (Barth et al., 1990; Jones et al., 1999) and because there were no significant effects of training on these measures in either sham or lesion groups.

\section{MAP2 immunocytochemistry}

On day 20 after lesions, animals in experiment 2 were given a lethal dose of sodium pentobarbitol and perfused intracardially with $\sim 200 \mathrm{ml}$ of 0.1 M phosphate buffer and $200 \mathrm{ml}$ of $4 \%$ paraformaldehyde in the same buffer. Brains were removed and stored for $1-3 \mathrm{~d}$ in fixative. Rostral-tocaudal sets of $50 \mu \mathrm{m}$ coronal sections through the sensorimotor cortex were obtained using a vibratome. Sections were then cryoprotected and stored at $-20^{\circ} \mathrm{C}$ until used. One set was stained with Toluidine Blue O (a Nissl stain) and used for the lesion verification, and an adjacent set was used for MAP2 immunocytochemistry.

A free-floating section immunocytochemistry method was used that previous studies have shown to produce good penetration of relatively thick sections $(50 \mu \mathrm{m})$ with evident high specificity and low background (Hawrylak and Greenough, 1995; Bury et al., 2000a). Sections were placed in $0.3 \% \mathrm{H}_{2} \mathrm{O}_{2}$ in $0.01 \mathrm{M}$ PBS at room temperature for $30 \mathrm{~min}$ to exhaust endogenous peroxidase activity. Sections were then rinsed in PBS and placed for $2 \mathrm{hr}$ at room temperature in a solution to block nonspecific protein binding. This block solution consisted of $0.2 \%$ Triton $\mathrm{X}-100$ and $0.1 \%$ bovine serum albumin in PBS with $2 \%$ horse serum. After rinses in PBS, sections were incubated at $4^{\circ} \mathrm{C}$ for $72 \mathrm{hr}$ in monoclonal anti-MAP2 (clone AP20, 1:1000 biotinylated anti-mouse IgG made in horse; Sigma, St. Louis, MO) in block solution. This antibody recognizes high molecular weight forms of MAP2 $(2 a+2 b)$ and is insensitive to phosphorylation state. Sections were then rinsed in PBS and placed for $1 \mathrm{hr}$ at room temperature in secondary antibody (1:200 biotinylated anti-rabbit IgG made in goat; Sigma) with $2 \%$ serum in PBS. After incubation, sections were again rinsed and incubated for $1 \mathrm{hr}$ in peroxidase-linked avidin-biotin complex (ABC kit, Vector Labs, Burlingame, CA). Immunoreactivity (IR) was visualized using standard 3-3' diaminobenzidine with nickel ammonium sulfate intensification procedures. Tissue from all groups was included in each batch of immunocytochemical processing. To verify specificity of antibody labeling, each batch of immunocytochemical processing included tissue sections processed without primary antibody (no-primary controls). These sections contained no evidence of distinctive process staining.

It is important to note that although changes in dendritic MAP2-IR are likely to indicate dendritic cytoskeletal changes, and net increases in MAP2-IR would be expected to accompany major dendritic growth (Philpot et al., 1997) (for review, see Itoh et al., 1997; Sánchez et al., 2000), changes in MAP2-IR alone are not revealing of the specific nature of the dendritic change. MAP2 stabilizes dendritic microtubules by forming longitudinal bridges between individual microtubule protofilaments (Al-Bassam et al., 2002), and it is believed to be a major target of intracellular signaling pathways and neurotrophins in the activitydependent formation of dendrites (Audesirk et al., 1997; Vaillant et al., 2002). In a previous study using this antibody, peak increases in MAP2-IR in layer V of the cortex opposite unilateral FLsmc lesions (Monahan et al., 2000) were found at time points (18-25 d) corresponding to time points of increased dendritic arborization of Golgi-Cox impregnated neurons and in dendrites visualized using electron microscopy within this region (Jones and Schallert, 1992; Jones et al., 1996).

\section{Quantification of MAP2 immunoreactive dendrites}

The cycloid grid intersection method (Baddeley et al., 1986) was used for measures of surface density ( $\mathrm{Sv}$ ) of MAP2-IR dendritic processes on tissue that was coded to conceal the experimental condition. MAP2-IR somata were excluded from these measurements. A set of test lines (cycloid arcs) arrayed in a complex, staggered manner was superimposed on light microscopic images. The number of intersections between immunoreactive dendritic processes and cycloid arcs was counted. The surface density was then calculated using the formula $\mathrm{Sv}=2(I / L)$, where $I$ is the total number of intersections and $L$ is the total test line length within the sample area. Within layers V and II/III of the agranular region of the FLsmc, 12 samples per layer (three samples per coronal section $\sim 250 \mu \mathrm{m}$ apart, from four sections within the sensorimotor cortex) were chosen using a systematic random sampling method and were visualized using a $100 \times$ oil immersion objective $(1680 \times$ final magnification). In all samples, the vertical axis of the cycloid arc test grid was aligned parallel to the orientation of cortical columns, defined as the orientation of the apical dendritic shafts of pyramidal neurons (i.e., apical shaft orientation was used to define local vertical windows) (Baddeley et al., 1986). The hemisphere measured was opposite the trained forelimb and/or opposite the unilateral lesion. In the Sham + No-Training group, the hemisphere measured was chosen randomly. To assess the specificity of the training and lesion effects to the motor cortex, samples were taken from layers II/III and V of the granular insular cortex, which is found lateral and ventral to the motor cortex within the same coronal sections. Six samples per layer were taken. This region is responsive to thermal stimulation of the tongue (Kosar et al., 1986), and previous studies have indicated a lack of dendritic and astroglial changes in this region after either transcallosal denervation or forced forelimb use (Bury et al., 2000a,b). Animals that had received postoperative preferred forelimb training were not included in the MAP2-IR analyses.

\section{Statistical analyses}

For reach-training experiments and behavioral tests, SPSS (SPSS, Inc.) general linear model (GLM) procedures for two-way ANOVA for groups and days were used. Post hoc comparisons of groups were performed when appropriate using SPSS procedure for contrasts. For the analysis of MAP2-IR and the lidocaine challenge data, SPSS GLM procedure for contrasts was used to perform planned comparisons to test the following: (1) and (2) whether training after FLsmc lesions changed MAP2-IR or 


\section{Experiment $1 \quad$ Experiment 2}

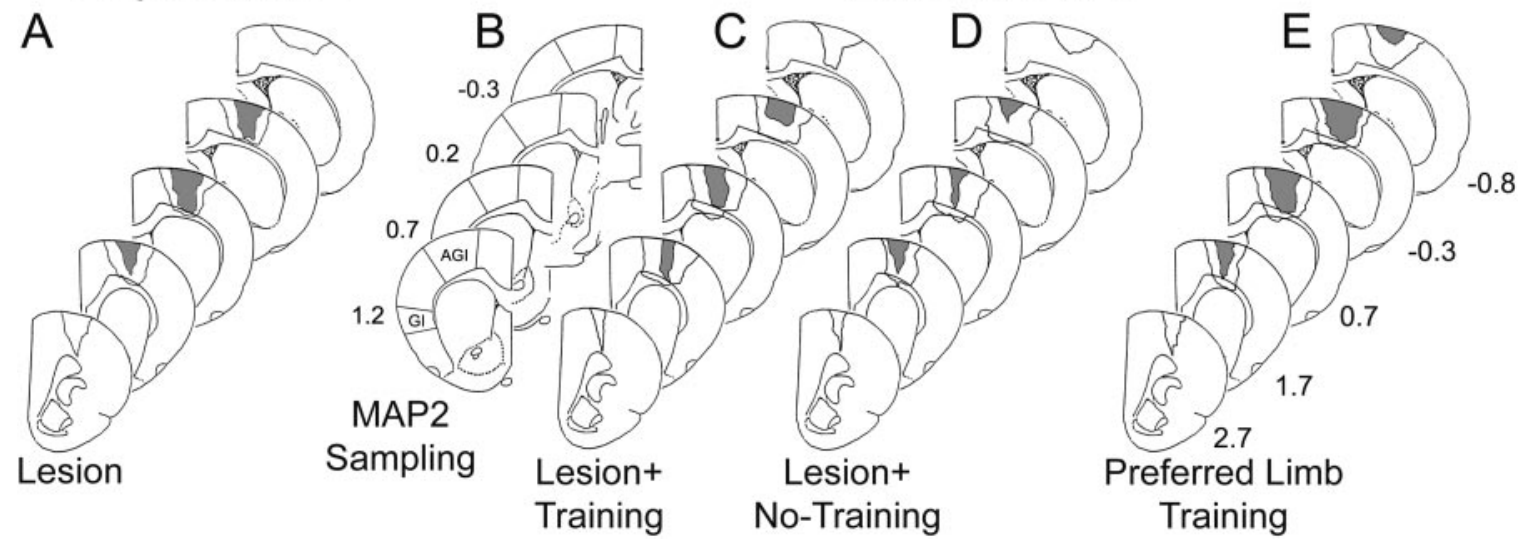

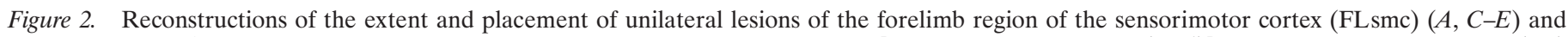

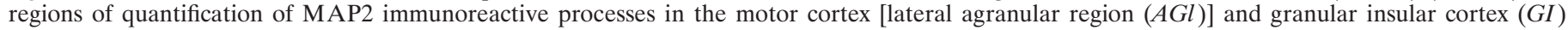

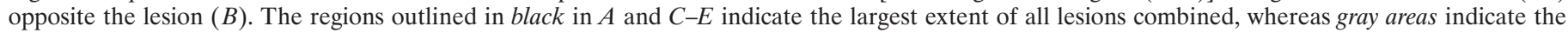
damaged tissue common to all lesions in that group. Numbers in $B$ and $E$ are approximate coordinates in millimeters relative to bregma.

affected the footfault test in comparison with either independent manipulation (Lesion + Training versus Lesion + No-Training and Lesion + Training versus Sham + Training), (3) whether lesions alone (Lesion + No-Training versus Sham + No-Training), and (4) whether reach training alone (Sham + Training versus Sham + No-Training) changed MAP2-IR or affected use of the forelimbs on the footfault test.

\section{RESULTS}

\section{Lesion extent and placement}

On the basis of lesion reconstructions using Nissl-stained coronal sections, all lesions included in this study appeared to produce major damage to the overlapping region of the forelimb sensorimotor cortex (Fig. 2). [In the rat, the caudal forelimb sensorimotor cortex consists of nonoverlapping primary somatosensory (SI) and primary motor $(\mathrm{MI})$ representation regions as well as a cytoarchitecturally distinctive MI-SI overlap zone (Donoghue and Wise, 1982)]. In experiment 1 , half of the animals exhibited near complete absence of a discernable overlap zone, whereas the remaining three had only a small lateral portion of the overlap area remaining. Two lesions in this experiment extended into the underlying white matter; however the striatum appeared intact. In experiment 2 , most animals also had a nearly a complete absence of the overlap zone $(n=5$ Lesion + Training; $n=4$ Lesion + No-Training; $n=3$ Lesion + Preferred Forelimb Training), whereas the remaining lesions appeared to have small lateral or medial portions of the overlap zone present. Several lesions also produced white matter damage $(n=3$ Lesion + Training; $n=3$ Lesion + No-Training; $n=2$ Lesion + Preferred Forelimb Training), and a subset of these produced minor, superficial damage to the dorsal striatum ( $n=1$ Lesion + Training; $n=2$ Lesion + No-Training; $n=1$ Preferred Forelimb Training). No differences in behavior or immunoreactivity could be traced to these differences in lesion extent or placement. One animal (not reported in the numbers above) in the Lesion + No-Training group was removed from the study because of an anterior lesion that failed to damage a significant portion of the overlap zone.

\section{Lesions improve acquisition of a skilled-reaching task with the nonimpaired forelimb}

Figure $3 A$ shows the performance with the preferred forelimb on the reaching task as assessed before surgery in experiment 1 . Both pre-lesion and pre-sham animals demonstrated increased reach- ing success over days of training and showed similar acquisition rates. Two-way ANOVA for the effects of group by day revealed a significant effect for day $\left(F_{(19,171)}=26.37 ; p<0.001\right)$, reflecting the improvement over days of training. However, there was no significant effect for group $\left(F_{(1,10)}=0.01 ; p>0.05\right)$, nor was there a significant effect for group by day $\left(F_{(19,171)}=0.61 ; p>0.05\right)$.

Figure $3 B$ shows the mean percentage of successful reaches with the nonpreferred limb in these same animals after either lesion or sham surgical procedures. Lesion animals made more successful reaches with their nonimpaired forelimb in comparison with shams [two-way ANOVA effects or group $\left(F_{(1,10)}=8.18\right.$; $p<0.05)]$. There was also a significant effect for day $\left(F_{(17,136)}=\right.$ 21.8; $p<0.001)$, as well a significant group by day interaction $\left(F_{(17,136)}=3.64 ; p<0.001\right)$, indicating a difference between lesion and sham animals in the rates of acquisition of the reaching task. Post hoc analyses revealed that lesion rats made significantly more successful reaches with the nonpreferred forelimb on most postsurgical days than sham animals. When the task was made more difficult, by moving the pellet farther away (day 6), both groups demonstrated lowered reaching success. By day 9, however, lesion animals were performing as well as they did with the close pellet trials, whereas sham animals had little further improvement in performance. Increasing the pellet size had little effect, resulting in only a very minor and transient reduction in reaching success in both groups.

It should be noted that one animal in the sham group never learned to reach successfully with its nonpreferred limb, demonstrating an unusually poor performance relative to the other sham-operated animals. However, removing this animal's data from the statistical analyses did not change the inferences. Group $\left(F_{(1,9)}=196.4 ; p<0.001\right)$, day $\left(F_{(17,119)}=25.84 ; p<0.001\right)$, and group by day interaction $\left.\left(F_{(17,119}\right)=3.40 ; p<0.001\right)$ effects for the percentage of successful reaches remained significant.

\section{Extensive preoperative training is not required for this effect}

Figure 4 shows the mean percentage of successful reaches for lesion and sham animals that received postoperative training with the nonpreferred forelimb in the absence of extensive preoperative training with the preferred limb (experiment 2). In close pellet training, rats with lesions showed a significantly greater rate 

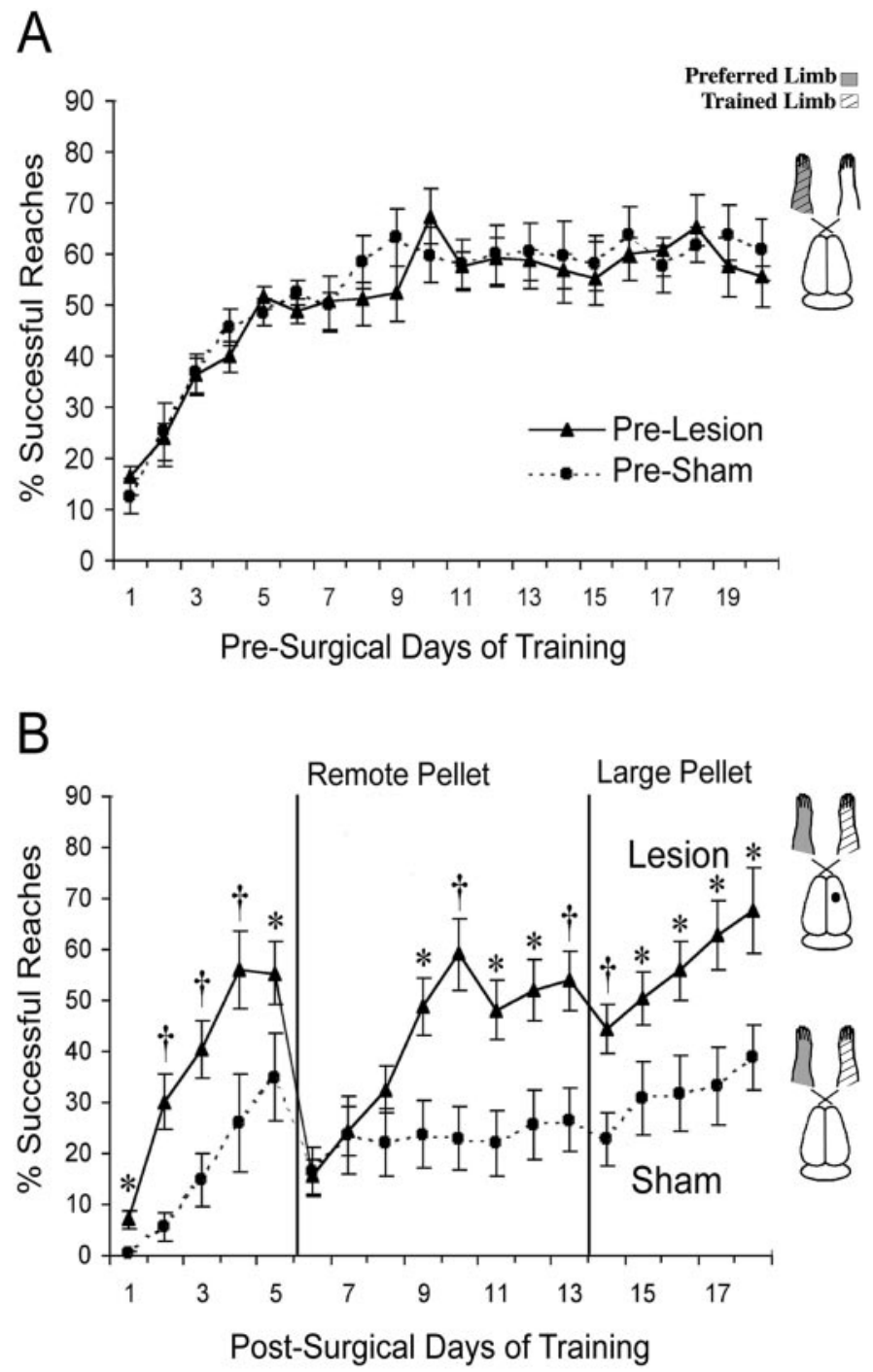

Figure 3. Successful reaches with the preferred forelimb on the pellet retrieval task for each group before surgery $(A)$ and with the previously nonpreferred limb after unilateral FLsmc lesions or sham operations $(B)$. The trained limb in animals with unilateral lesions was the nonimpaired forelimb (ipsilateral to the lesion). Lesion animals performed significantly better than shams on most postlesion days of training. On more difficult, remote pellet trials, rats with lesions rapidly acquired a proficiency equivalent to the performance in retrieving close pellets, whereas sham animals had more gradual and subtle improvements over days of training. To the right of the graphs are schematic illustrations of rat brains and the side of the lesion and the training relative to the preoperatively preferred forelimb. Data are means \pm SEM percentage of successful reaches/total reach attempts. ${ }^{*} p<0.05,{ }^{\dagger} p<0.01$ significantly different from shams.

of acquisition on the task compared with shams, although shams did eventually reach lesion levels of performance. On close pellet days of training, there was a significant effect for group $\left(F_{(1,14)}=\right.$ $13.5 ; p<0.01)$, for day $\left(F_{(12,168)}=106.1 ; p<0.001\right)$, and a group by day interaction $\left(F_{(12,168)}=2.24 ; p<0.05\right)$. When task difficulty was increased (remote pellet trials), both groups showed an initial drop in performance. However, by the second day of remote pellet training, lesion rats were significantly better at the task in comparison with shams and remained improved relative to shams throughout the remaining days of testing. For remote pellet trials, there was a significant effect of group $\left(F_{(1,14)}=262.5 ; p<0.05\right)$

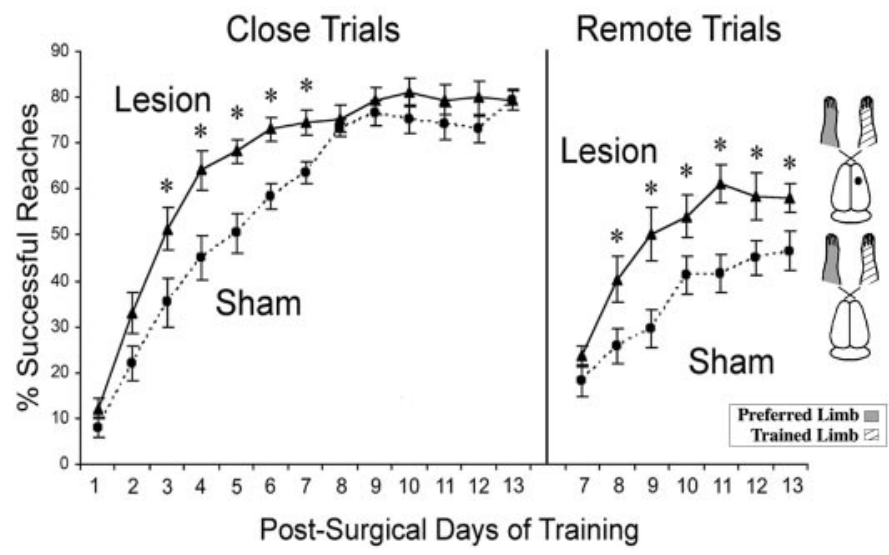

Figure 4. The percentage of successful reaches on the pellet retrieval task after unilateral FLsmc lesions or sham operations when no extensive training with the other limb was provided before surgery (i.e., experiment 2 ). The postsurgical training was with the preoperatively nonpreferred limb and in animals with unilateral FLsmc lesions, the nonimpaired limb (see illustrations to the right). Rats with unilateral FLsmc lesions demonstrated a faster rate of acquisition on the task in comparison with their sham counterparts on both close and remote pellets trails. Data are means \pm SEM. ${ }^{*} p<0.05$ significantly different from shams.

and day $\left(F_{(6,84)}=64.5 ; p<0.001\right)$ and a significant group by day interaction $\left(F_{(6,84)}=2.90 ; p<0.05\right)$.

\section{Facilitated "reversal of handedness" does not fully account for the improved performance}

Given previous research showing that unilateral motor cortex ablation reverses preferences for use of the limb opposite the lesion (Castro, 1977; Castro-Alamancos and Borrell, 1995), it seemed possible that the FLsmc lesions simply made rats more motivated to begin reaching with their nonimpaired (previously nonpreferred) limb than sham animals. Although the inner chamber wall and pellet placement prevented reach attempts with the previously preferred forelimb from being successful, all rats initially attempted to retrieve pellets with this limb. If sham animals made a greater number of unsuccessful attempts with the previously preferred forelimb, this could contribute to their inferior performance in comparison with the lesion group. In the first experiment, although lesions tended to result in a more rapid reversal of handedness, evidenced by a diminishment in reach attempts with the previously preferred limb (Fig. $5 A$ ), the use of this limb was highly variable within groups, and the lesion group was not significantly different in comparison with shams (two-way ANOVA effects of group: $F_{(1,10)}=18.8, p=0.71$; group by day: $\left.F_{(10,80)}=1.44, p=0.30\right)$. Within the first week after surgery and before the onset of more difficult (remote) reaching trials, both groups had complete reversal of handedness on this task.

In experiment 2 , there was also no significant difference between groups in the use of the previously preferred forelimb (two-way ANOVA effects of group: $F_{(1,14)}=2.92, p=0.21$; group by day: $F_{(4,56)}=0.07, p=0.90$ ) (Fig. $5 B$ ). Thus, although it remains possible that a greater reversal of handedness contributes to the greater rate of acquisition in the earlier days of training, it cannot explain the overall greater performance in the lesions versus shams. It should be noted that sham animals in experiment 2 tended to make fewer reach attempts with the previously preferred limb in comparison with shams of experiment 1 in the early days of postoperative training, indicating that extensive training with the preferred limb may increase the difficulty of reversing handedness. 


\section{A}

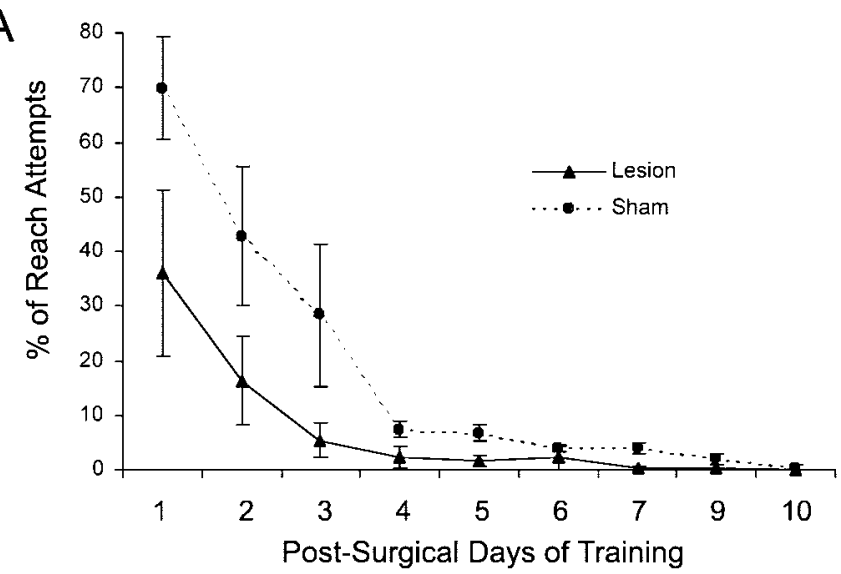

B

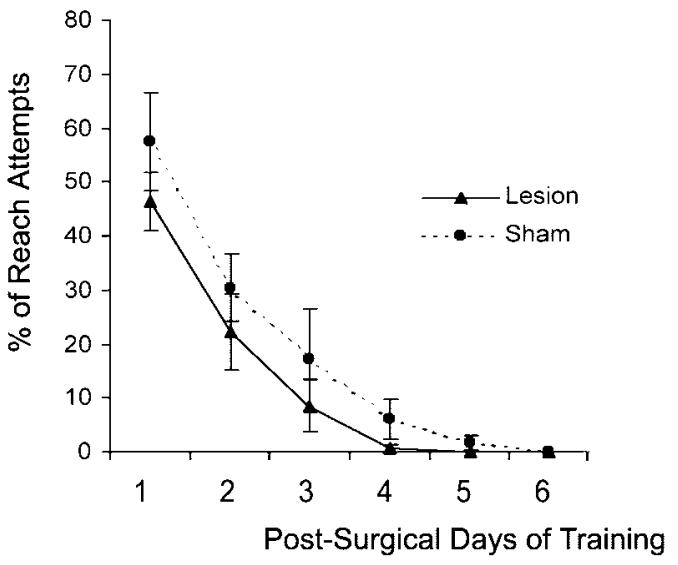

Figure 5. The percentage of reach attempts made with the previously preferred forelimb during postsurgical training in animals from experiment $1(A)$ and experiment $2(B)$. The design of the apparatus prevented reach attempts with this limb from being successful. Particularly in the first $3 \mathrm{~d}$ of training, sham animals with extensive preoperative training of the preferred forelimb (experiment $1, A$ ) tended to make more reach attempts with this limb than the lesion group of this experiment and both groups of experiment $2(B)$ that had no extensive presurgical training. However, there was not a significant group or group by day interaction effect on two-way ANOVA in either experiment. Data are means \pm SEM.

\section{The effect may not be dependent on endogenous limb preferences}

Data from a subset of animals ( $n=4$ per group) indicates that the enhancement of skilled reaching of the nonimpaired limb after unilateral FLsmc lesions is unlikely to require that this coincides with forced reversal of handedness, provided that the task is made sufficiently difficult. In this subset of rats, the trained limb was the preoperatively preferred limb. As with the previous experiments, in rats with lesions, the trained limb was also the nonimpaired limb. On remote pellet trials, lesion animals tended to perform better than shams. Over the first $7 \mathrm{~d}$ of training on close pellet trials, the mean \pm SEM percentage of successful reaches was $57.3 \pm 2.90$ for shams and $64.5 \pm 0.75$ for lesion rats. On remote pellet trials (days 7-12), the mean percentage of successful reaches was $40.2 \pm 2.85$ for shams and $53.1 \pm 4.70$ for lesion rats. Although there were no significant overall group or group by day interaction effects in reaching performance in this small subset of animals, performance on remote reaching trials approached significance (group effect: $F_{(1,6)}=30.6, p=0.057$ ). In both groups, performance tended to be better in comparison with animals trained with the nonpreferred limb, especially during the first
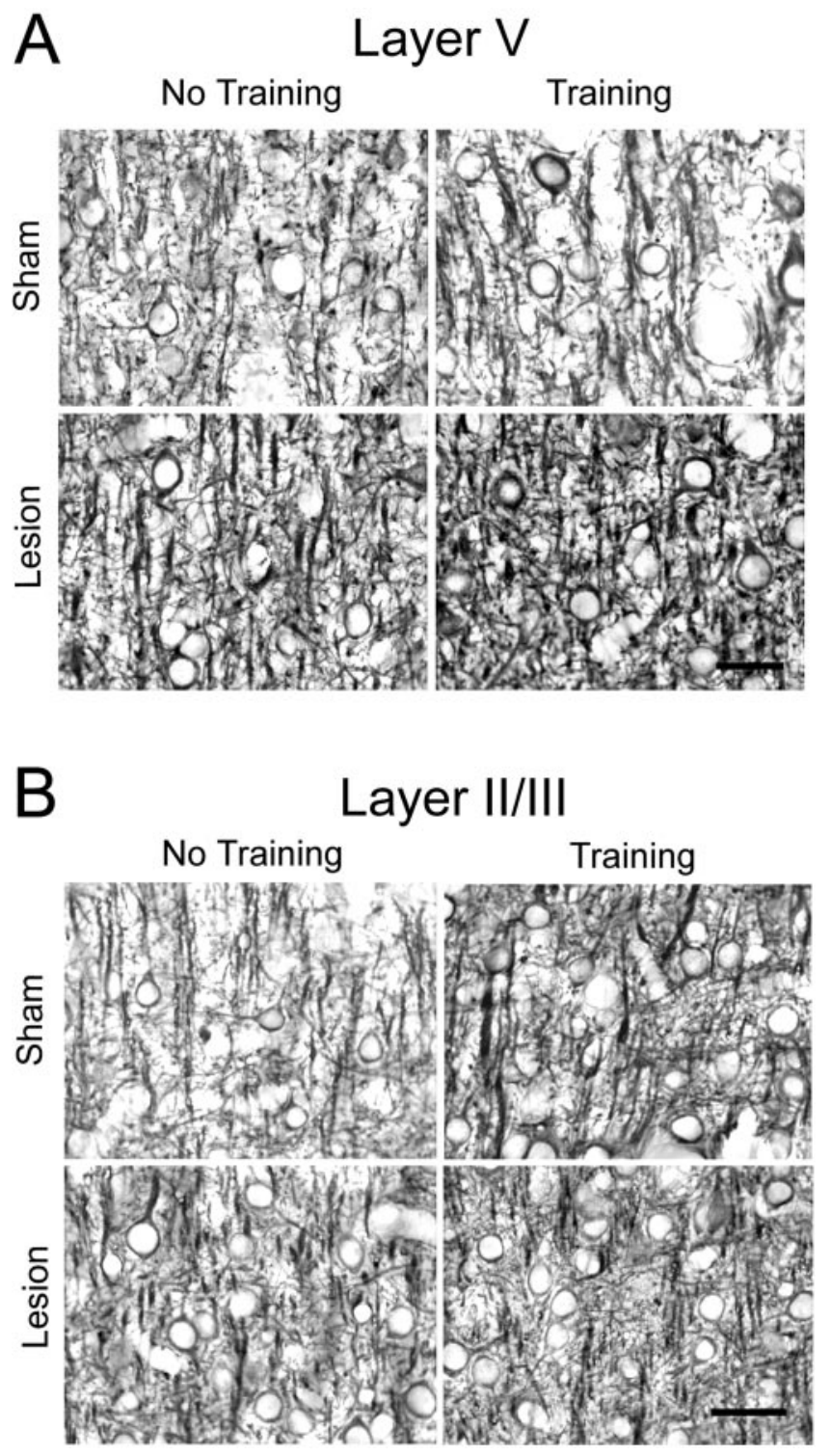

Figure 6. Representative photomicrographs of MAP2-IR dendrites in layer $\mathrm{V}(A)$ and layer II/III $(B)$ of the motor cortex opposite unilateral lesions and opposite the trained forelimb. Quantitative results are shown in Figure 7. Scale bar, $25 \mu \mathrm{m}$.

week of training. This is consistent with the likelihood that acquisition of skilled reaching is less challenging with the preferred limb than with the nonpreferred limb.

\section{Training-induced dendritic MAP2 changes are enhanced in layer $\mathbf{V}$ of the cortex opposite FLsmc lesions}

As shown in Figures $6 A$ and $7 A$, reach training in animals with unilateral FLsmc lesions (Lesion + Training) resulted in a significant increase in the surface density of MAP2-IR dendritic processes in layer $\mathrm{V}$ of the motor cortex opposite the lesions and trained forelimb in comparison with either manipulation alone (versus Sham + Training: $F_{(1,27)}=33.9, p<$ 0.001 ; versus Lesion + No-Training: $\left.F_{(1,27)}=7.05, p<0.05\right)$. Less robust, but significant, increases were found as a result of training in sham animals in comparison with Sham + NoTraining $\left(F_{(1,27)}=13.7 ; p<0.01\right)$. For rats that received reach 

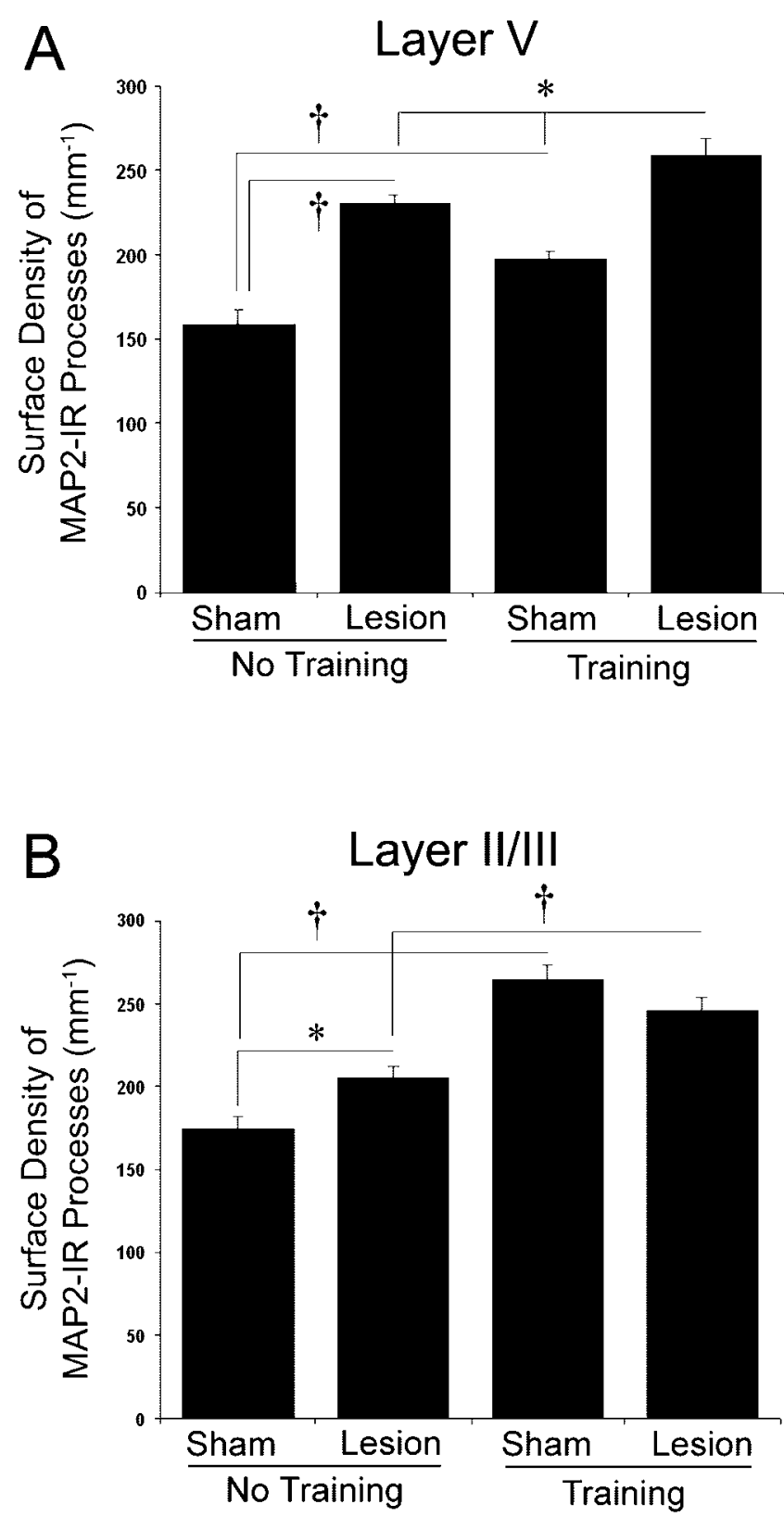

Figure 7. The surface density of MAP2-immunoreactive dendrites in layer $\mathrm{V}(A)$ and layer II/III $(B)$ of the motor cortex opposite the lesion and/or trained limb. In layer V, increases in MAP2-IR were especially evident after unilateral lesions, although there were also significant increases after reach training in intact animals (Sham + Training). Reach training after lesions (Lesion + Training) produced further increases in MAP2-IR dendrites than that found after lesions alone or training alone. In Layer II/III $(B)$, increases in the surface density of MAP2-IR dendrites were greatest in intact animals receiving unilateral reach training, whereas lesions alone produced a smaller but significant increase in comparison with Sham + No-Training. Reach training after the lesions increased MAP2-IR in comparison with lesions alone, but not in comparison with training alone in layer II/III. Data are means \pm SEM. * $p<$ $0.05 ;{ }^{\dagger} p<0.01$.

training, there was a significant positive correlation between the percentage of successful reaches (pooled across days) and the surface density of MAP2-IR in layer V (Pearson's $r=+0.61 ; p<$ 0.02 ). In the absence of training, unilateral lesions also resulted in a major increase in layer V MAP2-IR in comparison with Sham + No-Training $\left(F_{(1,27)}=43.0 ; p<0.001\right)$.

\section{Training and lesion-induced changes in MAP2-IR are laminar dependent}

As shown in Figures $6 B$ and $7 B$, the pattern of lesion- and training-induced MAP2-IR increases in layer II/III was different from that found in layer V. Training alone (Sham + Training) resulted in a greater increase in the surface density of MAP2-IR processes in comparison with Sham + No-Training in layer II/III than it did in layer V. Lesions alone more moderately, but significantly, increased the surface density of layer II/III MAP2-IR dendritic processes in comparison with Sham + NoTraining $\left(F_{(1,27)}=6.70 ; p<0.05\right)$. Although the combination of lesions and reach training did not further increase layer II/III MAP2-IR in comparison with training alone, MAP2-IR in the Lesion + Training group was significantly increased in comparison with lesions alone $\left(F_{(1,27)}=11.0 ; p<0.01\right)$. Layer II $/ \mathrm{III}$ MAP2-IR was not significantly correlated with the percentage of successful reaches (Pearson's $r=-0.20 ; p>0.05$ ). Together with the results from layer $\mathrm{V}$ (Fig. 7A), it appears that layer II/III is most affected by training alone, whereas the enhancement of the training-induced MAP2-IR changes is specific to layer V.

\section{Absence of MAP2-IR changes in the granular insular cortex}

In the granular insular cortex, which is found in the same coronal sections as those used for the motor cortical measures, the surface density of MAP2-IR processes in layers V and II/III were not significantly different between groups. The mean \pm SEM surface density of MAP2-IR processes in Sham + No-Training, Sham + Training, Lesion + No-Training, and Lesion + Training was $166 \pm 5.64,168 \pm 7.50,175 \pm 7.14$, and $167 \pm 10.87 \mathrm{~mm}^{-1}$ in layer V, respectively, and $177 \pm 4.99,170 \pm 9.05,180 \pm 6.61$, and $175 \pm 7.30 \mathrm{~mm}^{-1}$ in layer II/III, respectively. These results indicate that lesion and motor skills training effects on MAP2-IR are not generalized to the entire contralateral cortex.

\section{Training and lesion effects on tests of coordinated forelimb movements}

Figure 8 shows that the performance on the footfault test with the forelimb opposite the lesion was worsened as a result of the lesion, consistent with previous findings (Jones and Schallert, 1992; Jones et al., 1999). Both lesion groups had significant increases in forelimb errors per step with the impaired limb soon after surgery in comparison with shams but showed major improvement over days of testing. Two-way ANOVA indicated a significant group $\left(F_{(3,27)}=35.4 ; p<0.001\right)$, day $\left(F_{(1,27)}=45.2\right.$; $p<0.001)$, and group by day interaction effect $\left(F_{(1,27)}=15.5 ; p<\right.$ $0.001)$. Although there were no significant differences between the two lesion groups on post hoc comparisons, animals that had undergone the reach training tended to show slightly more errors on this task than the Lesion + No-Training group on days 8 and 18. In the Lesion + Training, but not in the Lesion + NoTraining group, the error rate remained significantly different from shams on days 8 and 18 on post hoc comparisons. There were no significant effects on two-way ANOVA of the percentage errors made with the ipsilateral/trained forelimb.

Anesthetization of the nonimpaired/trained limb (the lidocaine challenge) was performed to test the reliance on this limb on the footfault test. The assumption is that greater reliance on this forelimb will result in greater impairments on the footfault test when this limb is anesthetized (Schallert et al., 1997). Anesthetization of the nonimpaired limb resulted in a significant increase in errors made by the impaired forelimb in both lesion 


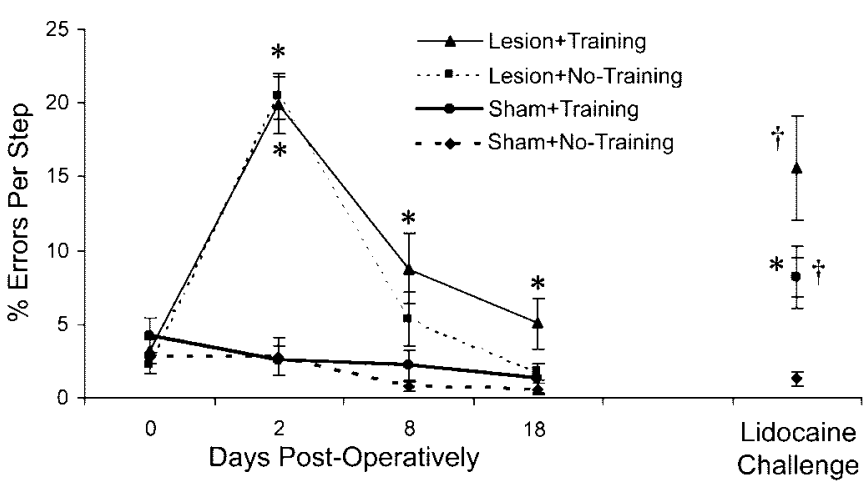

Figure 8. The percentage of errors per step taken with the nontrained and contralateral-to-the-lesion forelimb while wandering the grid floor of the footfault test. Errors were misplacements of the forepaw that resulted in slips through the grid openings. Animals with unilateral FLsmc lesions demonstrated an initial dramatic impairment in placements with the contralateral forelimb in comparison with shams that improved over time. Although there were no significant differences between the two lesion groups, rats receiving postlesion reach training with the nonimpaired forelimb (Lesion + Training) remained significantly different from shams on days 8 and 18, whereas Lesion + No-Training returned to nonsignificant levels in comparison with the shams by day 8 . On day 19, animals were observed on the footfault test with the nonimpaired/trained forelimb anesthetized (Lidocaine Challenge), which tests the reliance on this limb for traversing the grid floor. This resulted in greater errors with the impaired limb in both lesion groups. In the Lesion + Training and Sham + Training groups, errors with the nontrained limb were increased relative to animals of the same lesion condition that had not undergone reach training, suggesting that the reach training resulted in an increased reliance on the trained limb for the task. Data are means \pm SEM. ${ }^{*} p<$ 0.05 significantly different from sham of the same training condition. ${ }^{\dagger} p<$ 0.05 significantly different from the No-Training group of the same lesion condition.

groups (Lesion + Training vs Sham + Training: $F_{(1,27)}=5.60$, $p<0.05$; Lesion + No-Training vs Sham + No-Training: $\left.F_{(1,27)}=4.62, p<0.05\right)$, suggesting that improvements with the impaired limb on this task were likely to be caused, in part, by compensatory reliance on the nonimpaired limb, consistent with previous findings (Schallert et al., 1997). Furthermore, in both lesion and sham animals, the increase in error rate with anesthetization of the trained limb was greater than the nontrained controls of the same lesion condition (Lesion + Training vs Lesion + No-Training: $F_{(1,27)}=5.27, p<0.05$; Sham + Training vs Sham + No-Training: $\left.F_{(1,27)}=4.92, p<0.05\right)$. Thus, reach training may result in an increased reliance on the trained limb on this task, even in intact animals.

\section{DISCUSSION}

Unilateral lesions of the FLsmc increased the ability of adult rats to learn a skilled motor task with their nonimpaired forelimb in comparison with controls. Task difficulty was important in detecting these differences, whereas the ease of reversing handedness did not fully account for the enhanced learning. Moreover, these animals had enhanced dendritic plasticity in the motor cortex in comparison with training- or lesions-alone, as evidenced by increased surface density of MAP2-IR dendrites, consistent with the hypothesis that a lesion-induced enhancement of motor cortical neural plasticity is linked to the behavioral improvements. Different layers of the cortex seemed to be particularly sensitive to different aspects of the experimental manipulations (lesion versus forelimb training), and the MAP2 changes were found in a functionally relevant region of the motor cortex but not in a nearby cortical region, the granular insular cortex. Finally, training focused on the intact forelimb did not generalize to improvements on a task of bilateral coordinated motor behavior.

\section{Enhancement of motor skill learning with the nonimpaired forelimb}

A striking finding of the present study is that for a task that intact animals appear to find extremely challenging (remote pellet trials), FLsmc lesion rats are able to learn relatively as rapidly as they do with an easier task. The lesion-induced enhancement of skill acquisition was most apparent when the animals were required to switch to using a nonpracticed, nonpreferred limb after extensive preoperative training with the preferred limb. However, the lesion-induced enhancement was also evident in the absence of extensive preoperative training and is unlikely to be dependent on endogenous limb preferences.

This effect is probably not simply a result of a lesion-induced release of inhibition in the opposite motor cortex. Inhibition of the FLsmc opposite the preferred forelimb, using intracerebral injections of potassium chloride (Martin and Webster, 1974) or tetrodotoxin (Miklyaeva and Bures, 1991), does not result in an enhancement of the ability to reach with the nonpreferred forelimb. Thus, transcortical connections are unlikely to be normally confounding of unilateral reaching ability. [Transcallosal projections are predominantly excitatory and terminate on glutamatergic neurons (Buhl and Singer, 1989; Hughes and Peters, 1992).] The present findings seem likely to be related, at least in part, to an enhancement of training-induced neuroplastic changes in the motor cortex opposite the lesions.

\section{Training- and lesion-induced dendritic cytoskeletal plasticity in the motor cortex}

In intact animals, unilateral reach training resulted in a major increase in the surface density of MAP2-IR dendrites in the motor cortex opposite the trained limb, especially within layer II/III but also evident in layer V. This result was not surprising given previous findings that reach training in adult animals results in more and longer dendritic processes (Withers and Greenough, 1989) and increases in synapse number per neuron in the motor cortex contralateral to the trained limb (Kleim et al., 2002). The synaptic increases have been found to be concordant with changes in cortical representations of forelimb movements, revealed using microstimulation mapping (Kleim et al., 1998, 2002) (see also Nudo et al., 1996) and may be related to the behavioral induction of long-term potentiation in layer II/III (Rioult-Pedotti et al., 1998, 2000). Rats trained on a complex motor skills task (the acrobatic task) also show dramatic increases in synapses per neuron in the motor cortex (Kleim et al., 1996; Jones et al., 1999).

The finding of increased MAP2-IR in the cortex opposite unilateral FLsmc lesions is consistent with previous findings of major dendritic growth and synapse addition in the motor cortex opposite the lesion (Jones and Schallert, 1992; Jones et al., 1996, 1999; Jones, 1999). The postlesion dendritic structural changes have been linked to the combined effects of lesion-induced degeneration of transcallosal afferents and forelimb behavioral changes (Jones and Schallert, 1994; Bury et al., 2000a). Animals with unilateral FLsmc lesions spontaneously develop an increased reliance on the nonimpaired forelimb, and the neuronal structural changes in the opposite motor cortex occur in the absence of any additional behavioral manipulations. However, it is possible to enhance these structural changes using relevant behavioral manipulations, such as complex motor skills training, which has 
previously been found to enhance FLsmc lesion-induced synapse addition in the motor cortex (Jones et al., 1999). Biernaskie and Corbett (2001) have also found that dendritic growth in the cortex opposite unilateral middle cerebral artery occlusions is increased by postlesion complex rehabilitative experience. Consistent with these findings, in the present study, increases in MAP2-IR in the cortex opposite FLsmc lesions were further increased in animals undergoing unilateral reach training.

We have hypothesized previously that the enhanced neuroplastic response to behavioral change in the cortex opposite unilateral FLsmc lesions is, at least in part, a result of denervation-induced promotion of neuronal plasticity (Jones et al., 1999; Bury et al., 2000a). In several systems, denervation has been found to initiate growth-promoting processes including increases in neurotrophic factors, reactive changes in glial cells, and neuronal cytoskeletal restructuring (Cotman et al., 1981; Sheppard et al., 1993; Ridet et al., 1997; Steward and Trimmer, 1997). Although these changes support neural adaptation to lost afferents, they may also provide an exceptionally fertile substrate for neural restructuring and thus could facilitate some types of behavioral change. Denervation of transcallosal fibers results in astrocytic reactivity and increased glial expression of neurotrophic factors in the motor cortex (Gomide and Chadi, 1999; Bury et al., 2000b). Previously, we found that at least some of the astrocytic reactions to transcallosal denervation are enhanced by forced-use of the opposite forelimb (Bury et al., 2000a). It seems likely that similar denervationtriggered changes contribute to the enhancement of skilled reaching in the present study.

In addition to denervation-induced effects, it is possible that lesion-induced forelimb behavioral changes contribute to the enhancement of skilled reaching and MAP2-IR. Given that unilateral lesions cause greater reliance on the nonimpaired forelimb, it is possible that the greater home cage experience with the use of this limb facilitates skill acquisition on the reaching task. The extent to which denervation and behavioral experience contribute to the enhancement of skill learning would be addressed best using independent manipulations of these two factors, an important topic for future studies.

The laminar-dependent MAP2-IR changes observed in this study are consistent with previous findings of laminar differences in spine addition resulting from manipulations of denervation and forelimb use (Adkins et al., 2002). Forced-use of one forelimb in intact animals increased spine density in layer II/III pyramidal neuron dendrites, whereas denervation alone resulted in significant layers II/III and V spine addition. Denervation and forceduse together resulted in a further increase in spine density, but this additional increase was specific to layer $\mathrm{V}$ pyramidal neurons.

\section{Training effects on coordinated forelimb use}

Although the reach training failed to significantly affect other tests of sensorimotor function, in a bilateral task of coordinated forelimb use, the footfault test, reach training increased the reliance on the nonimpaired/trained forelimb in comparison with untrained animals. This increased reliance clearly did not result in an improved ability to accomplish this task and tended to worsen performance after the lesions. It is possible that the unilateral reach training resulted in a maladaptively extreme over-reliance on the nonimpaired forelimb for the bilateral coordination required for this task. In contrast, postlesion training on the acrobatic task, which requires the development of diverse bilateral coordinated movements, resulted in improvements in the use of both forelimbs on the footfault test (Jones et al., 1999).
Capitalizing on the increased capacity to learn motor skills with the nonimpaired forelimb to improve function seems likely to require coordinated training of this limb with the impaired limb.

\section{Implications for rehabilitative treatment}

These findings may have implications for the phenomenon of learned nonuse, as described in animals with peripheral sensory deafferentation and humans with unilateral strokes (for review, see Taub et al., 2002). Sensory deafferentation of one limb is believed to repeatedly reinforce the use of the nonimpaired limb in place of the impaired limb. The present findings suggest that learned nonuse is likely to be exaggerated after cortical damage in comparison with peripheral deafferentation. Further understanding of the relationship between learned nonuse and facilitated learning with the nonimpaired forelimb may help guide rehabilitative protocols, particularly therapies focused on coordinated use of the impaired with the nonimpaired limb.

The dependency of these effects on lesion size and postlesion time of training onset was not addressed directly in these studies. It is not known whether enhanced learning would be supported by a motor cortical region that is undergoing much greater degenerative and neurotoxic effects, such as might occur after much larger ischemic lesions, in contrast to the focal lesions of the present study. Nevertheless, even in a more severely disrupted motor cortex, the capacity to use behavioral manipulations to optimize neuronal adaptation seems likely to exist. Finally, given the well established time course of neural and glial events in the contralateral cortex after unilateral FLsmc lesions (Jones et al., 1998), the observed enhancement may be time dependent, i.e., there may a sensitive time window after the lesions when the motor cortex is most responsive to new motor skill learning.

\section{REFERENCES}

Adams FS, Schwarting RK, Huston JP (1994) Behavioral and neurochemical asymmetries following unilateral trephination of the rat skull: is this control operation always appropriate? Physiol Behav 55:947-952.

Adkins DL, Bury SD, Jones TA (2002) Callosal denervation and forelimb behavioral change result in laminar dependent dendritic and spine alterations in the motor cortex of adult rats. Neurobiol Learn Mem 78:35-52.

Al-Bassam J, Ozer RS, Safer D, Halpain S, Milligan RA (2002) MAP2 and tau bind longitudinally along the outer ridges of microtubule protofilaments. J Cell Biol 157:1187-1196.

Audesirk G, Cabell L, Kern M (1997) Modulation of neurite branching by protein phosphorylation in cultured rat hippocampal neurons. Dev Brain Res 102:247-260.

Baddeley AJ, Gundersen HJG, Cruz-Orvie LM (1986) Estimation of surface area from vertical sections. J Neurosci Methods 48:141-148.

Barth TM, Jones TA, Schallert T (1990) Functional subdivisions of the rat somatic-sensorimotor cortex. Behav Brain Res 39:73-95.

Biernaskie J, Corbett D (2001) Enriched rehabilitative training promotes improved forelimb motor function and enhanced dendritic growth after focal ischemic injury. J Neurosci 27:5272-5280.

Buhl EH, Singer W (1989) The callosal projection in cat visual cortex as revealed by a combination of retrograde tracing and intracellular injection. Exp Brain Res 75:470-476.

Bury SD, Adkins DL, Ishida JT, Kotzer CM, Eichhorn AC, Jones TA (2000a) Denervation facilitates neuronal growth in the motor cortex of rats in the presence of behavioral demand. Neurosci Lett 287:85-88.

Bury SD, Eichhorn AE, Kotzer CM, Jones TA (2000b) Reactive astrocytic responses to denervation in the motor cortex of adult rats are sensitive to manipulations of behavioral experience. Neuropharmacology 39:743-755.

Castro AJ (1977) Limb preference after lesions of the cerebral hemisphere in adult and neonatal rats. Physiol Behav 18:605-608.

Castro-Alamancos MA, Borrell J (1995) Functional recovery of forelimb response capacity after forelimb primary motor cortex damage in the rat is due to the reorganization of adjacent areas of cortex. Neuroscience 68:793-805.

Cotman CW, Nieto-Sampedro M, Harris EW (1981) Synapse replacement in the nervous system of adult vertebrates. Physiol Rev 61:684-784.

Dahms JM, Miller DE, Alford JL, Adkins DL, Jones TA (1999) The 
effects of motor skills training on astrocytic reactions to unilateral sensorimotor cortical damage in adult rats. Soc Neurosci Abstr 25:639.

Donoghue JP, Wise SP (1982) The motor cortex of the rat: cytoarchitecture and microstimulation mapping. J Comp Neurol 212:76-88.

Gomide VC, Chadi G (1999) The trophic factor S-100beta and basic fibroblast growth factor are increased in the forebrain reactive astrocytes of adult callosotomized rat. Brain Res 835:162-174.

Hawrylak N, Greenough WT (1995) Monocular deprivation alters the morphology of glial fibrillary acidic protein-immunoreactive astrocytes in the rat visual cortex. Brain Res 683:187-199.

Hughes CM, Peters A (1992) Symmetric synapses formed by callosal afferents in rat visual cortex. Brain Res 583:271-278.

Itoh TJ, Hisanaga S, Hosoi T, Kishimoto T, Hotani H (1997) Phosphorylation states of microtubule-associated protein 2 (MAP2) determine the regulatory role of MAP2 in microtubule dynamics. Biochemistry $36: 12574-12582$

Jones TA (1999) Multiple synapse formation in the motor cortex opposite unilateral sensorimotor cortex lesion in adult rats. J Comp Neurol 414:57-66.

Jones TA, Schallert T (1992) Overgrowth and pruning of dendrites in adult rats recovering from neocortical damage. Behav Brain Res $51: 156-160$.

Jones TA, Schallert T (1994) Use-dependent growth of pyramidal neurons after neocortical damage. J Neurosci 14:2140-2152.

Jones TA, Kleim JA, Greenough WT (1996) Neuronal growth contralateral to unilateral sensorimotor cortex damage in adult rats: a quantitative electron microscopic examination. Brain Res 733:142-148.

Jones TA, Hawrylak N, Klintsova AY, Greenough WT (1998) Brain damage behavior, rehabilitation, recovery, and brain plasticity. Ment Retard Dev Disabil Res Rev 4:231-237.

Jones TA, Chu CJ, Grande LA, Gregory AD (1999) Motor skills training enhances lesion-induced structural plasticity in the motor cortex of adult rats. J Neurosci 19:10153-10163.

Kleim JA, Lussnig E, Schwarz ER, Greenough WT (1996) Synaptogenesis and fos expression in the motor cortex of the adult rat following motor skill learning. J Neurosci 16:4529-4535.

Kleim JA, Barbay S, Nudo RJ (1998) Functional reorganization of the rat motor cortex following motor skill learning. J Neurophysiol 80:3321-3325.

Kleim JA, Barbay S, Cooper N, Hogg T, Reidel C, Remple M, Nudo RJ (2002) Motor-learning dependent synaptogenesis is localized to functionally reorganized motor cortex. Neurobiol Learn Mem 77:63-77.

Kobayashi N, Mundel PA (1998) Role of microtubules during the formation of cell processes in neuronal and non-neuronal cells. Cell Tissue Res 291:163-174.

Kosar E, Grill HJ, Norgren R (1986) Gustatory cortex in the rat I. Physiological properties and cytoarchitecture. Brain Res 379:329-341.

Martin D, Webster WG (1974) Paw preference shifts in the rat following forced practice. Physiol Behav 13:745-748.

McKenna JE, Whishaw IQ (1999) Complete compensation in skilled reaching success with associated impairments in limb synergies, after dorsal column lesion in the rat. J Neurosci 19:1885-1894.

Miklyaeva EI, Bures J (1991) Reversal of "handedness" in rats is achieved more effectively by training under peripheral than under central blockade of the preferred forepaw. Neurosci Lett 125:89-92.
Miklyaeva EI, Whishaw IO (1996) Hemi-Parkinson analogue rats display active support in good limbs versus passive support in bad limbs on a skilled reaching task of variable height. Behav Neurosci 110:117-125.

Monahan TM, Adkins DL, Jones TA (2000) Alterations in microtubuleassociated protein 2 in the cortex contralateral and homotopic to focal neocortical damage. Soc Neurosci Abstr 26:73.20.

Nudo RJ, Milliken GW, Jenkins WM, Merzenich M (1996) Usedependent alterations of movement representations in primary motor cortex of adult squirrel monkeys. J Neurosci 16:785-807.

Peterson GM, Devine JV (1963) Transfer on handedness in the rat resulting from small cortical lesions after limited forced practice. J Comp Physiol Psychol 56:752-756.

Philpot BD, Lim JH, Halpain S, Brunjes PC (1997) Experiencedependent modifications in MAP2 phosphorylation in rat olfactory bulb. J Neurosci 17:9596-9604.

Ridet JL, Malhorta SK, Privat A, Gage FH (1997) Reactive astrocytes: cellular and molecular cues to biological function. Trends Neurosci 20:570-577.

Rioult-Pedotti MS, Friedman D, Hess G, Donoghue JP (1998) Strengthening of horizontal cortical connections following skill learning. Nat Neurosci 1:230-234

Rioult-Pedotti MS, Friedman D, Donoghue JP (2000) Learning-induced LTP in neocortex. Science 290:533-536.

Sánchez C, Díaz-Nido J, Avila J (2000) Phosphorylation of microtubuleassociated protein 2 (MAP2) and its relevance for the regulation of the neuronal cytoskeleton function. Prog Neurobiol 61:133-168.

Schallert T, Whishaw IQ (1984) Bilateral cutaneous stimulation of the somatosensory system in hemi-decorticate rats. Behav Neurosci 98: $518-540$.

Schallert T, Upchurh M, Wilcox RE, Vaughn DM (1983) Posture independent sensorimotor analysis of inter-hemispheric receptor asymmetries in the neostriatum. Pharmacol Biochem Behav 18:753-759.

Schallert T, Kozlowski DA, Humm JL, Cocke RR (1997) Usedependent structural events in recovery of function. Adv Neurol 73:229-238

Sheppard AM, Wu JE, Staubli U, Perlmutter LS (1993) Changes in calpain and brain spectrin immunoreactivity accompany sprouting in the deafferented hippocampus. Synapse 15:239-242.

Steward O, Trimmer PA (1997) Genetic influences on cellular reactions to CNS injury: the reactive response of astrocytes in denervated neuropil regions in mice carrying a mutation $(\mathrm{WLD}(\mathrm{S}))$ that causes delayed Wallerian degeneration. J Comp Neurol 380:70-81.

Taub E, Uswatte G, Elbert T (2002) New treatments in neurorehabilitation founded on basic research. Nat Rev Neurosci 3:228-236.

Vaillant AR, Zanassi P, Walsh GS, Aumont A, Alonso A, Miller FD (2002) Signaling mechanisms underlying reversible, activity-dependent dendrite formation. Neuron 34:985-998.

Whishaw IQ (1992) Lateralization and reaching skill related: results and implications from a large sample of Long-Evans rats. Behav Brain Res 52:45-48.

Withers GS, Greenough WT (1989) Reach training selectively alters dendritic branching in subpopulations of Layer II/III pyramids in rat motor-somatosensory forelimb cortex. Neuropsychologia 27:61-69. 